\title{
The Thermodynamics in Planck's Law
}

\author{
Constantinos Ragazas ${ }^{1}$ \\ The Lawrenceville School
}

USA

\section{Introduction}

Quantum Physics has its historical beginnings with Planck's derivation of his formula for blackbody radiation, more than one hundred years ago. In his derivation, Planck used what latter became known as energy quanta. In spite of the best efforts at the time and for decades later, a more continuous approach to derive this formula had not been found. Along with Einstein's Photon Hypothesis, the Quantization of Energy Hypothesis thus became the foundations for much of the Physics that followed. This physical view has shaped our understanding of the Universe and has resulted in mathematical certainties that are counterintuitive and contrary to our experience.

Physics provides mathematical models that seek to describe what is the Universe. We believe mathematical models of what is -- as with past metaphysical attempts -- are a never ending search getting us deeper and deeper into the 'rabbit's hole' [Frank 2010]. We show in this Chapter that a quantum-view of the Universe is not necessary. We argue that a world without quanta is not only possible, but desirable. We do not argue, however, with the mathematical formalism of Physics -- just the physical view attached to this.

We will present in this Chapter a mathematical derivation of Planck's Law that uses simple continuous processes, without needing energy quanta and discrete statistics. This Law is not true by Nature, but by Math. In our view, Planck's Law becomes a Rosetta Stone that enables us to translate known physics into simple and sensible formulations. To this end the quantity eta we introduce is fundamental. This is the time integral of energy that is used in our mathematical derivation of Planck's Law. In terms of this prime physis quantity eta (acronym for energy-time-action), we are able to define such physical quantities as energy, force, momentum, temperature and entropy. Planck's constant $h$ (in units of energy-time) is such a quantity eta. Whereas currently $h$ is thought as action, in our derivation of Planck's Law it is more naturally viewed as accumulation of energy. And while $h$ is a constant, the quantity eta that appears in our formulation is a variable. Starting with eta, Basic Law can be mathematically derived and not be physically posited.

Is the Universe continuous or discrete? In my humble opinion this is a false dichotomy. It presents us with an impossible choice between two absolute views. And as it is always the case, making one side absolute leads to endless fabrications denying the opposite side. The Universe is neither continuous nor discrete because the Universe is both continuous and discrete. Our view of the Universe is not the Universe. The Universe simply is. In The Interaction of Measurement [Ragazas, 2010h] we argue with mathematical certainty that we

\footnotetext{
${ }^{1}$ cragaza@lawrenceville.org
} 
cannot know through direct measurements what a physical quantity $E(t)$ is as a function of time.

Since we are limited by our measurements of 'what is', we should consider these as the beginning and end of our knowledge of 'what is'. Everything else is just 'theory'. There is nothing real about theory! As the ancient Greeks knew and as the very word 'theory' implies. In Planck's Law is an Exact Mathematical Identity [Ragazas 2010f] we show Planck's Law is a mathematical truism that describes the interaction of measurement. We show that Planck's Formula can be continuously derived. But also we are able to explain discrete 'energy quanta'. In our view, energy propagates continuously but interacts discretely. Before there is discrete manifestation we argue there is continuous accumulation of energy. And this is based on the interaction of measurement.

Mathematics is a tool. It is a language of objective reasoning. But mathematical 'truths' are always 'conditional'. They depend on our presuppositions and our premises. They also depend, in my opinion, on the mental images we use to think. We phrase our explanations the same as we frame our experiments. In the single electron emission double-slit experiment, for example, it is assumed that the electron emitted at the source is the same electron detected at the screen. Our explanation of this experiment considers that these two electrons may be separate events. Not directly connected by some trajectory from source to sensor. [Ragazas 2010j]

We can have beautiful mathematics based on any view of the Universe we have. Consider the Ptolemy with their epicycles! But if the view leads to physical explanations which are counter-intuitive and defy common sense, or become too abstract and too removed from life and so not support life, than we must not confuse mathematical deductions with physical realism. Rather, we should change our view! And just as we can write bad literature using good English, we can also write bad physics using good math. In either case we do not fault the language for the story. We can't fault Math for the failings of Physics.

The failure of Modern Physics, in my humble opinion, is in not providing us with physical explanations that make sense; a physical view that is consistent with our experiences. A view that will not put us at odds with ourselves, with our understanding of our world and our lives. Math may not be adequate. Sense may be a better guide.

\section{Mathematical results}

We list below the main mathematical derivations that are the basis for the results in physics in this Chapter. The proofs can be found in the Appendix at the end. These mathematical results, of course, do not depend on Physics and are not limited to Physics. In Stocks and Planck's Law [Ragazas 20101] we show how the same 'Planck-like' formula we derive here also describes a simple comparison model for stocks.

Notation.

$E(t)$ is a real-valued function of the real-variable $t$

$\tau=\Delta t=t-s$ is an interval of $t$

$\Delta E=E(t)-E(s)$ is the change of $E$

$\eta=P=\int_{s}^{t} E(u) d u$ is the accumulation of $E$

$\bar{E}=E_{a v}=\frac{1}{t-s} \int_{s}^{t} E(u) d u$ is the average of $E$ 
$\mathscr{J}=\mathcal{T}_{\eta}=\left(\frac{1}{\kappa}\right) \frac{\eta}{\tau}$ where $\kappa$ is a scalar constant

$D_{x}$ indicates differentiation with respect to $x$

$r, v$ are constants, often a rate of growth or frequency

Characterization 1: $\quad E(t)=E_{0} e^{r t}$ if and only if $\Delta E=P r$

Characterization 2: $\quad E(t)=E_{0} e^{r t}$ if and only if $\frac{P r}{e^{r(t-s)}-1}=E(s)$

Characterization 2a: $\quad E(t)=E_{0} e^{r t}$ if and only if $\frac{P r}{e^{P r / E_{a v}}-1}=E(s)$

Characterization 3: $\quad E(t)=E_{0} e^{r t}$ if and only if $\frac{\Delta E}{e^{\Delta E / E_{a v}}-1}=E(s)$

Characterization 4: $\quad E(t)=E_{0} e^{r t}$ if and only if $\frac{\Delta E}{E_{a v}}=r \Delta t$

Theorem 1a: $\quad E(t)=E_{0} e^{r t}$ if and only if $\frac{P r}{e^{P r / E_{a v}}-1}$ is invariant with $t$

Theorem 2: $\quad$ For any integrable function $E(t), \lim _{t \rightarrow s} \frac{P r}{e^{r \Delta t}-1}=E(s)$

\section{1 'Planck-like' characterizations [Ragazas 2010a]}

Note that $E_{a v}=\kappa \mathcal{T}_{\eta}$. We can re-write Characterization $2 a$ above as,

$$
E(t)=E_{0} e^{v t} \quad \text { if and only if } \quad E_{0}=\frac{\eta v}{e^{\eta v / \kappa \mathcal{T}}-1}
$$

Planck's Law for blackbody radiation states that, $\quad E_{0}=\frac{h v}{e^{h v / k T}-1}$

where $E_{0}$ is the intensity of radiation, $v$ is the frequency of radiation and $T$ is the (Kelvin) temperature of the blackbody, while $h$ is Planck's constant and $k$ is Boltzmann's constant. [Planck 1901, Eqn 11]. Clearly (1) and (2) have the exact same mathematical form, including the type of quantities that appear in each of these equations. We state the main results of this section as,

Result I: A 'Planck-like' characterization of simple exponential functions

$$
E(t)=E_{0} e^{v t} \quad \text { if and only if } \quad E_{0}=\frac{\eta v}{e^{\eta v / \kappa \mathcal{T}}-1}
$$

Using Theorem 2 above we can drop the condition that $E(t)=E_{0} e^{v t}$ and get, Result II: A 'Planck-like' limit of any integrable function

$$
\text { For any integrable function } E(t), E_{0}=\lim _{t \rightarrow 0} \frac{\eta v}{e^{\eta v / \kappa \mathcal{T}}-1}
$$

We list below for reference some helpful variations of these mathematical results that will be used in this Chapter. 


$$
\begin{gathered}
\left.E_{0}=\frac{\Delta E}{e^{\Delta E / E_{a v}}-1}=\frac{\eta v}{e^{\eta v / \kappa \mathcal{F}_{\eta}}-1} \quad \text { (if } E(t)=E_{0} e^{v t}\right) \\
E_{0} \approx \frac{\Delta E}{e^{\Delta E / E_{a v}}-1} \approx \frac{\eta v}{e^{\eta v / \kappa \mathcal{T}_{\eta}}-1} \quad \text { (if } E(t) \text { is integrable) } \\
E_{0}=\frac{\eta v}{e^{\eta v / \kappa \mathcal{F}_{\eta}}-1} \text { is exact if and only if } \frac{\eta v}{e^{\eta v / \kappa \mathcal{F}_{\eta}}-1} \text { is independent of } \eta
\end{gathered}
$$

Note that in order to avoid using limit approximations in (4) above, by (3) we will assume an exponential of energy throughout this Chapter. This will allow us to explore the underlying ideas more freely and simply. Furthermore in Section $\mathbf{1 0 . 0}$ of this Chapter, we will be able to justify such an exponential time-dependent local representation of energy [Ragazas 2010i]. Otherwise, all our results (with the exception of Section 8.0) can be thought as pertaining to a blackbody with perfect emission, absorption and transmission of energy.

\section{Derivation of planck's law without energy quanta [Ragazas 2010f]}

Planck's Formula as originally derived describes what physically happens at the source. We consider instead what happens at the sensor making the measurement. Or, equivalently, what happens at the site of interaction where energy exchanges take place. We assume we have a blackbody medium, with perfect emission, absorption and transmission of energy. We consider that measurement involves an interaction between the source and the sensor that results in energy exchange. This interaction can be mathematically described as a functional relationship between $E(s)$, the energy locally at the sensor at time $s ; \Delta E$, the energy absorbed by the sensor making the measurement; and $\bar{E}$, the average energy at the sensor during measurement. Note that Planck's Formula (2) has the exact same mathematical form as the mathematical equivalence (3) and as the limit (4) above. By letting $E(s)$ be an exponential, however, from (3) we get an exact formula, rather than the limit (4) if we assume that $E(s)$ is only an integrable function. The argument below is one of several that can be made. The Assumptions we will use in this very simple and elegant derivation of Planck's Formula will themselves be justified in later Sections 5.0, 6.0 and 10.0 of this Chapter.

Mathematical Identity. For any integrable function $E(t), \quad \eta=\int_{s}^{s+\eta / E_{a v}} E(u) d u$

Proof: (see Fig. 1)

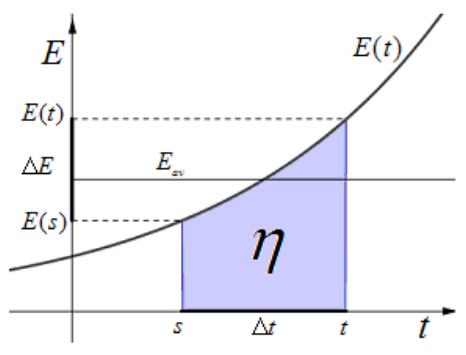

Fig. 1. 
Assumptions: 1) Energy locally at the sensor at $t=s$ can be represented by $E(s)=E_{0} e^{v s}$, where $E_{0}$ is the intensity of radiation and $v$ is the frequency of radiation. 2) When measurement is made, the source and the sensor are in equilibrium. The average energy of the source is equal to the average energy at the sensor. Thus, $\bar{E}=k T$. 3) Planck's constant $h$ is the minimal 'accumulation of energy' at the sensor that can be manifested or measured. Thus we have $\eta=h$.

Using the above Mathematical Identity (6) and Assumptions we have Planck's Formula,

$$
h=\int_{0}^{\frac{h}{k T}} E_{0} e^{v u} d u=\frac{E_{0}}{v}\left[e^{h v / k T}-1\right] \text { and so, } E_{0}=\frac{h v}{e^{h v / k T}-1}
$$

Planck's Formula is a mathematical truism that describes the interaction of energy. That is to say, it gives a mathematical relationship between the energy locally at the sensor, the energy absorbed by the sensor, and the average energy at the sensor during measurement. Note further that when an amount of energy $\Delta E$ is absorbed by the sensor, $E(t)$ resets to $E_{0}$.

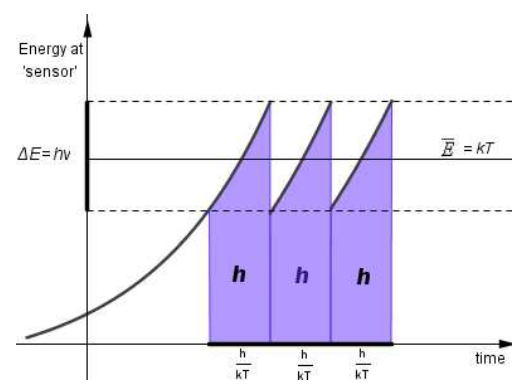

(a)

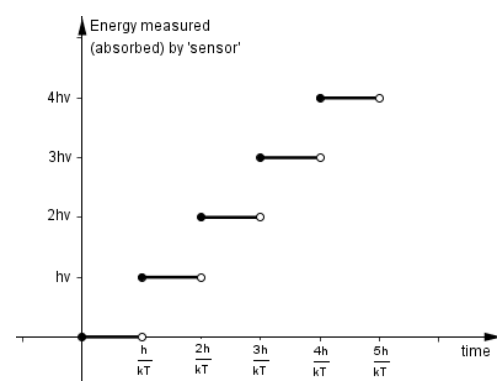

(b)

Fig. 2.

Note: Our derivation, showing that Planck's Law is a mathematical truism, can now clearly explain why the experimental blackbody spectrum is so indistinguishable from the theoretical curve. (http://en.wikipedia.org/wiki/File:Firas_spectrum.jpg)

Conclusions:

1. Planck's Formula is an exact mathematical truism that describes the interaction of energy.

2. Energy propagates continuously but interacts discretely. The absorption or measurement of energy is made in discrete 'equal size sips'(energy quanta).

3. Before manifestation of energy (when an amount $\Delta E$ is absorbed or emitted) there is an accumulation of energy that occurs over a duration of time $\Delta t$.

4. The absorption of energy is proportional to frequency, $\Delta E=h v$ (The Quantization of Energy Hypothesis).

5. There exists a time-dependent local representation of energy, $E(t)=E_{0} e^{\nu t}$, where $E_{0}$ is the intensity of radiation and $v$ is the frequency of radiation. [Ragazas 2011a]

6. The energy measured $\Delta E$ vs. $\Delta t$ is linear with slope $v k T$ for constant temperature $T$.

7. The time $\Delta t$ required for an accumulation of energy $h$ to occur at temperature $T$ is given by $\Delta t=\frac{h}{k T}$. 


\section{Prime physis eta and the derivation of Basic Law [Ragazas 2010d]}

In our derivation of Planck's Formula the quantity $\eta$ played a prominent role. In this derivation $\eta$ is the time-integral of energy. We consider this quantity $\eta$ as prime physis, and define in terms of it other physical quantities. And thus mathematically derive Basic Law. Planck's constant $h$ is such a quantity $\eta$, measured in units of energy-time. But whereas $h$ is a constant, $\eta$ is a variable in our formulation.

Definitions: For fixed $\left(\overrightarrow{\mathbf{x}}_{0}, t_{0}\right)$ and along the $x$-axis for simplicity, Prime physis : $\eta=$ eta (energytime-action)

$$
\begin{gathered}
\text { Energy: } \quad E=\frac{\partial \eta}{\partial t} \\
\text { Momentum: } p_{x}=\frac{\partial \eta}{\partial x} \\
\text { Force: } F_{x}=\frac{\partial^{2} \eta}{\partial x \partial t}
\end{gathered}
$$

Note that the quantity eta is undefined. But it can be thought as 'energy-time-action' in units of energy-time. Eta is both action as well as accumulation of energy. We make only the following assumption about $\eta$.

Identity of Eta Principle: For the same physical process, the quantity $\eta$ is one and the same.

Note: This Principle is somewhat analogous to a physical system being described by the wave function. Hayrani Öz has also used originally and consequentially similar ideas in [Öz 2002, 2005, 2008, 2010].

\subsection{Mathematical derivation of Basic Law}

Using the above definitions, and known mathematical theorems, we are able to derive the following Basic Law of Physics:

- $\quad$ Planck's Law, $E_{0}=\frac{h v}{e^{h v / k T}-1}$, is a mathematical truism (Section 3.0)

- The Quantization of Energy Hypothesis, $\Delta E=n h v \quad$ (Section 3.0)

- Conservation of Energy and Momentum. The gradient of $\eta(\overrightarrow{\mathbf{x}}, t)$ is $\vec{\nabla} \eta=\left\langle\frac{\partial \eta}{\partial x}, \frac{\partial \eta}{\partial t}\right\rangle=\left\langle p_{x}, E\right\rangle$. Since all gradient vector fields are conservative, we have the Conservation of Energy and Momentum.

- Newton's Second law of Motion. The second Law of motion states that $F=m a$. From definition (9) above we have,

$$
F=\frac{\partial^{2} \eta}{\partial x \partial t}=\frac{\partial^{2} \eta}{\partial t \partial x}=\frac{\partial p_{x}}{\partial t}=\frac{\partial}{\partial t}(m v)=m a, \text { since } p_{x}=m v
$$


- Energy-momentum Equivalence. From the definition of energy $E=\frac{\partial \eta}{\partial t}$ and of momentum $p_{x}=\frac{\partial \eta}{\partial x}$ we have that, $\eta=\int_{t_{0}}^{t} E(u) d u$ and $\eta=\int_{x_{0}}^{x} p_{x}(u) d u$.

Using the Identity of Eta Principle, the quantity $\eta$ in these is one and the same. Therefore, $\int_{t_{0}}^{t} E(u) d u=\int_{x_{0}}^{x} p_{x}(u) d u$. Differentiating with respect to $t$, we obtain, $E(t)=p_{x}(x) \cdot \frac{d x}{d t}$ or more simply, $E=p_{x} v$ (energy-momentum equivalence)

- Schroedinger Equation: Once the extraneous constants are striped from Schroedinger's equation, this in essence can be written as $\frac{\partial \psi}{\partial t}=H \psi$, where $\psi$ is the wave function, $H$ is the energy operator, and $H \psi$ is the energy at any $(\overrightarrow{\mathbf{x}}, t)$. The definition (7) of energy $\frac{\partial \eta}{\partial t}=E$ given above is for a fixed $\left(\overrightarrow{\mathbf{x}}_{0}, t_{0}\right)$. Comparing these we see that whereas our definition of energy is for fixed $\left(\overrightarrow{\mathbf{x}}_{0}, t_{0}\right)$, Schroedinger equation is for any $(\overrightarrow{\mathbf{x}}, t)$. But otherwise the two equations have the same form and so express the same underlying idea. Now (7) defines energy in terms of the more primary quantity $\eta$ (which can be viewed as accumulation of energy or action) and so we can view Schroedinger Equation as in essence defining the energy of the system at any $(\overrightarrow{\mathbf{x}}, t)$ while the wave function $\psi$ can be understood to express the accumulation of energy at any $(\overrightarrow{\mathbf{x}}, t)$. This suggest that the wave function $\psi$ is the same as the quantity $\eta$. We have the following interesting interpretation of the wave function.

- The wave function gives the distribution of the accumulation of energy of the system.

- Uncertainty Principle: Since $\Delta E=\eta v$, for $\Delta t>\frac{1}{v} \quad$ (a 'wavelength') we have $\Delta E \cdot \Delta t>\eta v \cdot \frac{1}{v}=\eta>h$. Or equivalently, for $\frac{\Delta E}{E_{a v}}>1$, we again have $\Delta E \cdot \Delta t>\eta>h$, since $h$ is the minimal eta that can be manifested. Note that since $\frac{\Delta E}{E_{a v}}=v \Delta t$ (Characteristic 5), we have $\frac{\Delta E}{E_{a v}}>1$ if and only if $\Delta t>\frac{1}{v}$. Since $E_{a v}=k T$ and entropy is defined as $\Delta S=\frac{\Delta E}{T}$, we have that

- $\Delta E \cdot \Delta t>h$ if and only if $\Delta S>k$.

- Planck's Law and Boltzmann's Entropy Equation Equivalence:

Starting with our Planck's Law formulation, $E_{0}=\frac{\Delta E}{e^{\Delta E / E_{a v}}-1}$ in (3) above and re-writing this equivalently we have, $e^{\Delta E / E_{a v}}=1+\frac{\Delta E}{E_{0}}=\frac{E}{E_{0}}$ and so, $\frac{\Delta E}{E_{a v}}=\ln \left(\frac{E}{E_{0}}\right)$. Using the definition of 
thermodynamic entropy we get $\Delta S_{\Theta}=\frac{\Delta E}{T}=k \cdot \frac{\Delta E}{E_{a v}}=k \cdot \ln \left(\frac{E}{E_{0}}\right)$. If $\Omega(t)$ represents the number of microstates of the system at time $t$, then $E(t)=A \Omega(t)$, for some constant $A$. Thus, we get Boltzmann's Entropy Equation, $S_{\Theta}=k \ln \Omega$.

Conversely, starting with Boltzmann's Entropy Equation, $\Delta S_{\Theta}=k \ln \left(\frac{\Omega}{\Omega_{0}}\right)=k \ln \left(\frac{E}{E_{0}}\right)$. Since $\Delta S_{\Theta}=\frac{\Delta E}{T}$ we can rewrite this equivalently as $\frac{\Delta E}{E_{a v}}=\ln \left(\frac{E}{E_{0}}\right)$ and so $e^{\Delta E / E_{a v}}=1+\frac{\Delta E}{E_{0}}=\frac{E}{E_{0}}$. From this we have, Planck's Law, $E_{0}=\frac{\Delta E}{e^{\Delta E / E_{a v}}-1}$ in (3) above.

- Entropy-Time Relationship: $\Delta S=k v \Delta t$ where $v$ is the rate of evolution of the system and $\Delta t$ is the time duration of evolution, since $E_{a v}=\frac{\eta}{\Delta t}$ and $\Delta E=\eta v$.

- The Fundamental Thermodynamic Relation: It is a well known fact that the internal energy $\mathrm{U}$, entropy $\mathrm{S}$, temperature $\mathrm{T}$, pressure $\mathrm{P}$ and volume $\mathrm{V}$ of a system are related by the equation $d U=T d S-P d V$. By using increments rather than differentials, and using the fact that work performed by the system is given by $W=\int P d V$ this can be re-written as $\Delta S=\frac{\Delta U}{T}+\frac{\Delta W}{T}$. All the terms in this equation are various entropy quantities. The fundamental thermodynamic relation can be interpreted thus as saying, "the total change of entropy of a system equals the sum of the change in the internal (unmanifested) plus the change in the external (manifested) entropy of the system". Considering the entropy-time relationship above, this can be rephrased more intuitively as saying "the total lapsed time for a physical process equals the time for the 'accumulation of energy' plus the time for the 'manifestation of energy' for the process". This relationship along with The Second Law of Thermodynamics establish a duration of time over which there is accumulation of energy before manifestation of energy - one of our main results in this Chapter and a premise to our explanation of the double-slit experiment. [Ragazas 2010j]

\section{The temperature of radiation [Ragazas 2010g]}

Consider the energy $E(t)$ at a fixed point at time $t$. We define the temperature of radiation to be given by $\mathcal{T}=\mathcal{T}_{\eta}=\left(\frac{1}{\kappa}\right) \frac{\eta}{\tau}$ where $\kappa$ is a scalar constant. Though in defining temperature this way the accumulation of energy $\eta$ can be any value, when considering a temperature scale $\eta$ is fixed and used as a standard for measurement. To distinguish temperature and temperature scale we will use $\mathcal{T}$ and $\mathcal{T}_{\eta}$ respectively. We assume that temperature is characterized by the following property:

Characterization of temperature: For a fixed $\eta$, the temperature is inversely proportional to the duration of time for an accumulation of energy $\eta$ to occur. 
Thus if temperature is twice as high, the accumulation of energy will be twice as fast, and visa-versa. This characterization of temperature agrees well with our physical sense of temperature. It is also in agreement with temperature as being the average kinetic energy of the motion of molecules.

For fixed $\eta$, we can define $\mathcal{T}_{\eta}=\left(\frac{1}{\kappa}\right) \frac{\eta}{\tau}$, which will be unique up to an arbitrary scalar constant $\kappa$. Conversely, for a given $\mathcal{T}$ as characterized above, we will have $\mathcal{T}=\rho \cdot \frac{1}{\tau}$, where $\rho$ is a proportionality constant. By setting $\rho=\frac{\eta}{\kappa}$ we get $\mathcal{T}=\mathcal{T}_{\eta}=\left(\frac{1}{\kappa}\right) \frac{\eta}{\tau}$. We have the following temperature-eta correspondence:

Temperature-eta Correspondence: Given $\eta$, we have $\mathcal{T}_{\eta}=\left(\frac{1}{\kappa}\right) \frac{\eta}{\tau}$, where $\kappa$ is some arbitrary scalar constant. Conversely, given $\mathcal{T}$ we have $\mathcal{T}=\mathcal{T}_{\eta}=\left(\frac{1}{\kappa}\right) \frac{\eta}{\tau}$, for some fixed $\eta$ and arbitrary scalar constant $\kappa$. Any temperature scale. therefore, will have some fixed $\eta$ and arbitrary scalar constant $\kappa$ associated with it.

\section{The meaning and existence of Planck's constant $h$ [Ragazas 2010c]}

Planck's constant $h$ is a fundamental universal constant of Physics. And although we can experimentally determine its value to great precision, the reason for its existence and what it really means is still a mystery. Quantum Mechanics has adapted it in its mathematical formalism. But QM does not explain the meaning of $h$ or prove why it must exist. Why does the Universe need $h$ and energy quanta? Why does the mathematical formalism of QM so accurately reflect physical phenomena and predict these with great precision? Ask any physicists and uniformly the answer is "that's how the Universe works". The units of $h$ are in energy-time and the conventional interpretation of $h$ is as a quantum of action. We interpret $h$ as the minimal accumulation of energy that can be manifested. Certainly the units of $h$ agree with such interpretation. Based on our results above we provide an explanation for the existence of Planck's constant -- what it means and how it comes about. We show that the existence of Planck's constant is not necessary for the Universe to exist but rather $h$ exists by Mathematical necessity and inner consistency of our system of measurements.

Using eta we defined in Section $\mathbf{5 . 0}$ above the temperature of radiation as being proportional to the ratio of eta/time. To obtain a temperature scale, however, we need to fix eta as a standard for measurement. We show below that the fixed eta that determines the Kelvin temperature scale is Planck's constant $h$.

In The Interaction of Measurement [Ragazas 2010h] we argue that direct measurement of a physical quantity $E(t)$ involves a physical interaction between the source and the sensor. For measurement to occur an interval of time $\Delta t$ must have lapsed and an incremental amount $\Delta E$ of the quantity will be absorbed by the sensor. This happens when there is an equilibrium between the source and the sensor. At equilibrium, the 'average quantity from the source' will equal to the 'average quantity $E_{a v}$ at the sensor'. Nothing in our observable World can exist without time, when the entity 'is' in equilibrium with its environment and its 'presence' can be observed and measured. Furthermore as we showed above in Section 3.0 the interaction of measurement is described by Planck's Formula. 
From the mathematical equivalence (5) above we see that $\eta$ can be any value and $\frac{\eta v}{e^{\eta v / \kappa \mathscr{S}_{\eta}}-1}$ will be invariant and will continue to equal to $E_{0}$. We can in essence (Fig. 3) 'reduce' the formula $E_{0}=\frac{\eta v}{e^{\eta v / \kappa \mathcal{J}_{\eta}}-1}$ by reducing the value of $\eta$ and so the value of $E_{a v}=\kappa \mathcal{T}_{\eta}$ will correspondingly adjust, and visa versa. Thus we see that $\eta$ and $\mathscr{T}_{\eta}$ go handin-hand to maintain $E_{0}=\frac{\eta v}{e^{\eta v / \kappa \mathcal{T}_{\eta}}-1}$ invariant. And though the mathematical equivalence (5) above allows these values to be anything, the calibrations of these quantities in Physics require their value to be specific. Thus, for $\eta=h$ (Planck's constant) and $\kappa=k$ (Boltzmann's constant), we get $\mathcal{T}_{\eta}=T$ (Kelvin temperature) (see Fig. 3). Or, conversely, if we start with $\mathcal{J}_{\eta}=T$ and set the arbitrary constant $\kappa=k$, then this will force $\eta=h$. Thus we see that Planck's constant $h$, Boltzmann's constant $k$, and Kelvin temperature $T$ are so defined and calibrated to fit Planck's Formula. Simply stated, when $\eta=h, \mathcal{T}_{h}=T$.
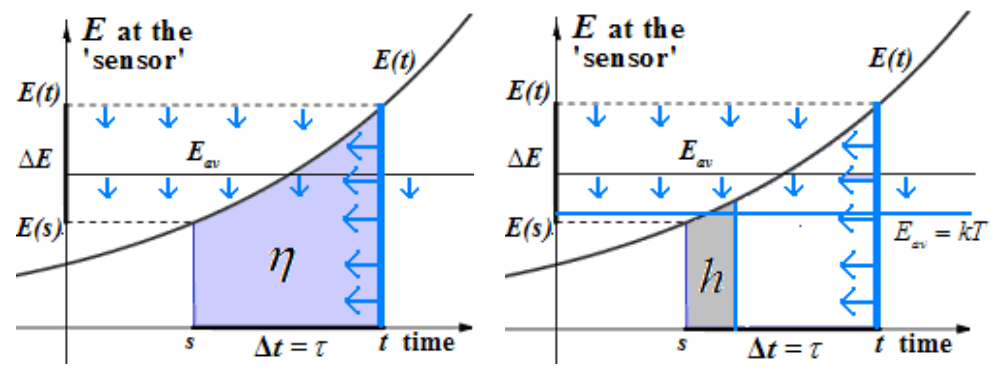

Fig. 3. $E_{0}=\frac{\Delta E}{e^{\Delta E / E_{a v-1}}}=\frac{\eta v}{e^{\eta v / \kappa \mathcal{T}} \eta_{-1}}, \Delta E=\eta v, E_{a v}=\kappa \mathcal{T}_{\eta}, \mathcal{T}_{\eta}=\left(\frac{1}{\kappa}\right) \frac{\eta}{\tau}, E(t)=E_{0} e^{v t}$

Conclusion: Physical theory provides a conceptual lens through which we 'see' the world. And based on this theoretical framework we get a measurement methodology. Planck's constant $h$ is just that 'theoretical focal point' beyond which we cannot 'see' the world through our theoretical lens. Planck's constant $h$ is the minimal eta that can be 'seen' in our measurements. Kelvin temperature scale requires the measurement standard eta to be $h$.

Planck's Formula is a mathematical identity that describes the interaction of measurement. It is invariant with time, accumulation of energy or amount of energy absorbed. Planck's constant exists because of the time-invariance of this mathematical identity. The calibration of Boltzmann's constant $k$ and Kelvin temperature $T$, with $k T$ being the average energy, determine the specific value of Planck's constant $h$.

\section{Entropy and the second law of thermodynamics [Ragazas 2010b]}

The quantity $\frac{\Delta E}{E_{a v}}$ that appears in our Planck's Law formulation (3) is 'additive over time'. This is so because under the assumption that Planck's Formula is exact we have that $\frac{\Delta E}{E_{a v}}=v \Delta t$, by 
Characterization 4. Interestingly, this quantity is essentially thermodynamic entropy, since $E_{a v}=k T$, and so $\Delta S=\frac{\Delta E}{T}=k v \Delta t$. Thus entropy is additive over time. Since $v$ can be thought as the evolution rate of the system (both positive or negative), entropy is a measure of the amount of evolution of the system over a duration of time $\Delta t$. Such connection between entropy as amount of evolution and time makes eminent intuitive sense, since time is generally thought in terms of change. But, of course, this is physical time and not some mathematical abstract parameter as in spacetime continuum.

Note that in the above, entropy can be both positive or negative depending on the evolution rate $v$. That the duration of time $\Delta t$ is positive, we argue, is postulated by The Second Law of Thermodynamics. It is amazing that the most fundamental of all physical quantities time has no fundamental Basic Law pertaining to its nature. We argue the Basic Law pertaining to time is The Second Law of Thermodynamics. Thus, a more revealing rewording of this Law should state that all physical processes take some positive duration of time to occur. Nothing happens instantaneously. Physical time is really duration $\Delta t$ (or $\mathrm{dt}$ ) and not instantiation $t=s$.

\section{The photoelectric effect without photons [Ragazas 2010k]}

Photoelectric emission has typically been characterized by the following experimental facts (some of which can be disputed, as noted):

1. For a given metal surface and frequency of incident radiation, the rate at which photoelectrons are emitted (the photoelectric current) is directly proportional to the intensity of the incident light.

2. The energy of the emitted photoelectron is independent of the intensity of the incident light but depends on the frequency of the incident light.

3. For a given metal, there exists a certain minimum frequency of incident radiation below which no photoelectrons are emitted. This frequency is called the threshold frequency. (see below)

4. The time lag between the incidence of radiation and the emission of photoelectrons is very small, less than $10^{-9}$ second.

Explanation of the Photoelectric Effect without the Photon Hypothesis: Let $v$ be the rate of radiation of an incident light on a metal surface and let $\alpha$ be the rate of absorption of this radiation by the metal surface. The combined rate locally at the surface will then be $v-\alpha$. The radiation energy at a point on the surface can be represented by $E(t)=E_{0} e^{(v-\alpha) t}$, where $E_{0}$ is the intensity of radiation of the incident light. If we let $\eta$ be the accumulation of energy locally at the surface over a time pulse $\tau$, then by Characterization 1 we'll have that $\Delta E=\eta(v-\alpha)$. If we let Planck's constant $h$ be the accumulation of energy for an electron, the number of electrons $n_{e}$ over the pulse of time $\tau$ will then be $n_{e}=\frac{\eta}{h}$ and the energy of an electron $\Delta E_{e}$ will be given by

$$
\Delta E_{e}=\frac{\Delta E}{n_{e}}=h(v-\alpha)
$$

Since $\eta=\int_{0}^{\tau} E_{0} e^{(v-\alpha) u} d u=E_{0}\left[\frac{e^{(v-\alpha) \tau}-1}{(v-\alpha)}\right]$, we get the photoelectric current $I$, 


$$
I=\frac{n_{e}}{\tau}=\frac{\eta}{h \tau}=E_{0}\left[\frac{e^{(v-\alpha) \tau}-1}{h(v-\alpha) \tau}\right]
$$

The absorption rate $\alpha$ is a characteristic of the metal surface, while the pulse of time $\tau$ is assumed to be constant for fixed experimental conditions. The quantity $\left[\frac{e^{(v-\alpha) \tau}-1}{h(v-\alpha) \tau}\right]$ in equation (10) would then be constant.

Combining the above and using (10) and (11) we have The Photoelectric Effect:

1. For incident light of fixed frequency $v$ and fixed metal surface, the photoelectric current $I$ is proportional to the intensity $E_{0}$ of the incident light. (by (11) above)

2. The energy $\Delta E_{e}$ of a photoelectron depends only on the frequency $v$ and not on the intensity $E_{0}$ of the incident light. It is given by the equation $\Delta E_{e}=h(v-\alpha)$ where $h$ is Planck's constant and the absorption rate $\alpha$ is a property of the metal surface. (by (10) above)

3. If $\Delta E_{e}$ is taken to be the kinetic energy of a photoelectron, then for incident light with frequency $v$ less than the 'threshold frequency' $\alpha$ the kinetic energy of a photoelectron would be negative and so there will be no photoelectric current. (by (10) above) (see Note below)

4. The photoelectric current is almost instantaneous $\left(<10^{-9} \mathrm{sec}\right)$, since for a single photoelectron we have that $\Delta t=\frac{h}{k T}<10^{-9}$ sec. by Conclusion 7 Section 3.

Note: Many experiments since the classic 1916 experiments of Millikan have shown that there is photoelectric current even for frequencies below the threshold, contrary to the explanation by Einstein. In fact, the original experimental data of Millikan show an asymptotic behavior of the (photocurrent) vs (voltage) curves along the energy axis with no clear 'threshold frequency'. The photoelectric equations (10) and (11) we derived above agree with these experimental anomalies, however.

In an article Richard Keesing of York University, UK, states,

I noticed that a reverse photo-current existed ... and try as I might I could not get rid of it.

My first disquieting observation with the new tube was that the I/V curves had high energy tails on them and always approached the voltage axis asymptotically. I had been brought up to believe that the current would show a well defined cut off, however my curves just refused to do so.

Several years later I was demonstrating in our first year lab here and found that the apparatus we had for measuring Planck's constant had similar problems.

After considerable soul searching it suddenly occurred on me that there was something wrong with the theory of the photoelectric effect ... [Keesing 2001]

In the same article, taking the original experimental data from the 1916 experiments by Millikan, Prof. Keesing plots the graphs in Fig. 4.

In what follows, we analyze the asymptotic behavior of equation (11) by using a function of the same form as (11).

$$
f(x)=\frac{A\left(e^{b(x-c)}-1\right)}{x-c}+d
$$

Note: We use d since some graphs typically are shifted up a little for clarity. 


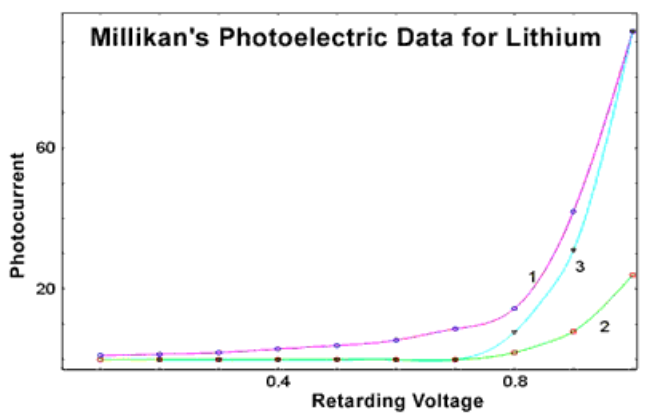

Fig. 4.

The graphs in Fig. 5 match the above experimental data to various graphs (in red) of equation (12)

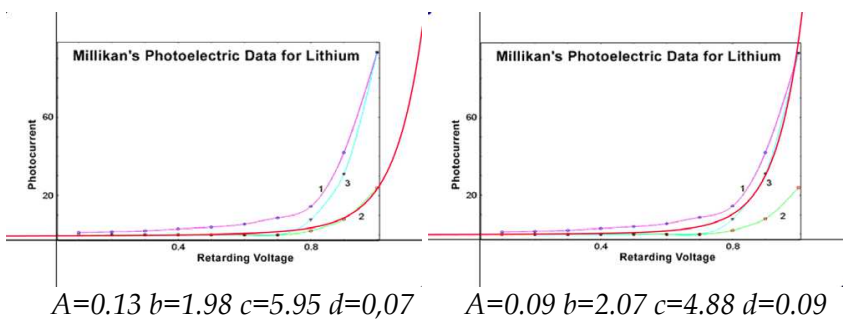

(a)

(b)

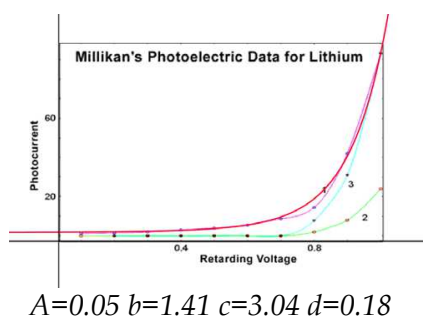

(c)

Fig. 5.

The above graphs (Fig. 5) seem to suggest that Eq. (11) agrees well with the experimental data showing the asymptotic behavior of the (photocurrent) v (energy) curves. But more systematic experimental work is needed.

\section{Meaning and derivation of the de broglie equations [Ragazas 2011a]}

Consider $\eta_{0}\left(x_{0}, t_{0}\right) \rightarrow \eta(x, t)$. We can write $\frac{\Delta \eta}{\eta_{0}}=\%$-change of $\eta=$ 'cycle of change'. For corresponding $\Delta x$ and $\Delta t$ we can write, $\lambda=\frac{\Delta x}{\Delta \eta / \eta_{0}}=$ "distance per cycle of change" and $\Delta \eta / \eta=\Delta \eta / \Delta t$ $v=\frac{\eta_{0}}{\Delta t}=$ "cycle of change per time". We can rewrite these as $\lambda=\frac{\eta_{0}}{\Delta \eta / \Delta x}$ and $v=\frac{\Delta \eta / \Delta t}{\eta_{0}}$. Taking limits and letting $\eta_{0}=h$ (Planck's constant being the minimal $\eta$ that can be measured) we get the de Broglie equations:

$$
\lambda=\frac{\eta_{0}}{\Delta \eta / \Delta x} \rightarrow \frac{h}{\partial \eta / \partial x}=\frac{h}{p} \text { and } v=\frac{\Delta \eta / \Delta t}{\eta_{0}} \rightarrow \frac{\partial \eta / \partial t}{h}=\frac{E}{h}
$$


Note: Since \%-change in $\eta$ can be both positive or negative, $\lambda$ and $v$ can be both positive or negative.

\section{The 'exponential of energy' $E(t)=E_{0} e^{v t}$ [Ragazas 2010i, 2011a]}

From Section 9.0 above we have that $v$ equals "\%-change of $\eta$ per unit of time". If we consider continuous change, we can express this as $\eta=\eta_{0} e^{\nu t}$. Differentiating with respect to $t$ we have, $E(t)=\frac{\partial \eta}{\partial t}=\eta_{0} v e^{v t}$ and $E_{0}=\eta_{0} v$. Thus, $E(t)=E_{0} e^{v t}$

\section{Proposition: "If the speed of light is constant, then light is a wave" [Ragazas 2011b]}

Proof: We have that $\lambda=\frac{h}{p}, v=\frac{E}{h}$ and $\lambda v=c$. Since $p=\frac{\partial \eta}{\partial x}$ and $E=\frac{\partial \eta}{\partial t}$, we have that

$$
\begin{aligned}
& \lambda v=\frac{\partial \eta / \partial t}{\partial \eta / \partial x} \text {. Differentiating, we get } \\
& \qquad D_{t}(\lambda v)=\frac{\frac{\partial^{2} \eta}{\partial t^{2}} \cdot \frac{\partial \eta}{\partial x}-\frac{\partial \eta}{\partial t} \cdot \frac{\partial^{2} \eta}{\partial t \partial x}}{\left(\frac{\partial \eta}{\partial x}\right)^{2}} \text { and } D_{x}(\lambda v)=\frac{\frac{\partial^{2} \eta}{\partial x \partial t} \cdot \frac{\partial \eta}{\partial x}-\frac{\partial \eta}{\partial t} \cdot \frac{\partial^{2} \eta}{\partial x^{2}}}{\left(\frac{\partial \eta}{\partial x}\right)^{2}}
\end{aligned}
$$

Since $\lambda v=c$, we have that $D_{t}(\lambda v)=0$ and $D_{x}(\lambda v)=0$. Therefore,

$$
\frac{\partial^{2} \eta}{\partial t^{2}} \cdot \frac{\partial \eta}{\partial x}-\frac{\partial \eta}{\partial t} \cdot \frac{\partial^{2} \eta}{\partial t \partial x}=0 \text { and } \frac{\partial^{2} \eta}{\partial x \partial t} \cdot \frac{\partial \eta}{\partial x}-\frac{\partial \eta}{\partial t} \cdot \frac{\partial^{2} \eta}{\partial x^{2}}=0
$$

Using $\lambda v=\frac{\partial \eta / \partial t}{\partial \eta / \partial x}$ and $\lambda v=c$, these can be written as,

$$
\frac{\partial^{2} \eta}{\partial t^{2}}=c \cdot \frac{\partial^{2} \eta}{\partial t \partial x} \quad \text { and } \quad \frac{\partial^{2} \eta}{\partial x \partial t}=c \frac{\partial^{2} \eta}{\partial x^{2}}
$$

Since 'mixed partials are equal', these equations combine to give us,

$$
\frac{\partial^{2} \eta}{\partial t^{2}}=c^{2} \cdot \frac{\partial^{2} \eta}{\partial x^{2}} \text {, the wave equation in one dimension }
$$

Thus, for the speed of light to be constant the 'propagation of light' $\eta$ must be a solution to the wave equation. q.e.d

\section{The double-slit experiment [Ragazas 2011a]}

The 'double-slit experiment' (where a beam of light passes through two narrow parallel slits and projects onto a screen an interference pattern) was originally used by Thomas Young in 
1803, and latter by others, to demonstrate the wave nature of light. This experiment later came in direct conflict, however, with Einstein's Photon Hypothesis explanation of the Photoelectric Effect which establishes the particle nature of light. Reconciling these logically antithetical views has been a major challenge for physicists. The double-slit experiment embodies this quintessential mystery of Quantum Mechanics.
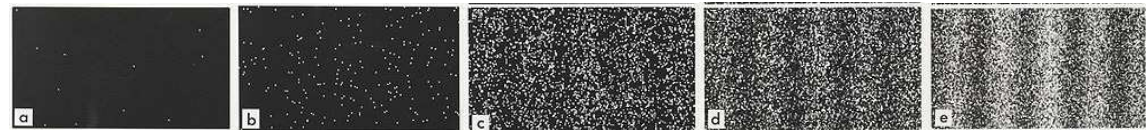

Fig. 6.

There are many variations and strained explanations of this simple experiment and new methods to prove or disprove its implications to Physics. But the 1989 Tonomura 'single electron emissions' experiment provides the clearest expression of this wave-particle enigma. In this experiment single emissions of electrons go through a simulated double-slit barrier and are recorded at a detection screen as 'points of light' that over time randomly fill in an interference pattern. The picture frames in Fig. 6 illustrate these experimental results. We will use these results in explaining the double-slit experiment.

\subsection{Plausible explanation of the double-slit experiment}

The basic logical components of this double-slit experiment are the 'emission of an electron at the source' and the subsequent 'detection of an electron at the screen'. It is commonly assumed that these two events are directly connected. The electron emitted at the source is assumed to be the same electron as the electron detected at the screen. We take the view that this may not be so. Though the two events (emission and detection) are related, they may not be directly connected. That is to say, there may not be a 'trajectory' that directly connects the electron emitted with the electron detected. And though many explanations in Quantum Mechanics do not seek to trace out a trajectory, nonetheless in these interpretations the detected electron is tacitly assumed to be the same as the emitted electron. This we believe is the source of the dilemma. We further adapt the view that while energy propagates continuously as a wave, the measurement and manifestation of energy is made in discrete units (equal size sips). This view is supported by all our results presented in this Chapter. And just as we would never characterize the nature of a vast ocean as consisting of discrete 'bucketfuls of water' because that's how we draw the water from the ocean, similarly we should not conclude that energy consists of discrete energy quanta simply because that's how energy is absorbed in our measurements of it.

The 'light burst' at the detection screen in the Tonomura double-slit experiment may not signify the arrival of "the" electron emitted from the source and going through one or the other of the two slits as a particle strikes the screen as a 'point of light'. The 'firing of an electron' at the source and the 'detection of an electron' at the screen are two separate events. What we have at the detection screen is a separate event of a light burst at some point on the screen, having absorbed enough energy to cause it to 'pop' (like popcorn at seemingly random manner once a seed has absorbed enough heat energy). The parts of the detection screen that over time are illuminated more by energy will of course show more 'popping'. The emission of an electron at the source is a separate event from the detection of a light burst at the screen. Though these events are connected they are not directly connected. There is no trajectory that connects these two electrons as being one and the same. The electron 'emitted' is not the same electron 'detected'. 
What is emitted as an electron is a burst of energy which propagates continuously as a wave and going through both slits illuminates the detection screen in the typical interference pattern. This interference pattern is clearly visible when a large beam of energy illuminates the detection screen all at once. If we systematically lower the intensity of such electron beam the intensity of the illuminated interference pattern also correspondingly fades. For small bursts of energy, the interference pattern illuminated on the screen may be undetectable as a whole. However, when at a point on the screen local equilibrium occurs, we get a 'light burst' that in effect discharges the screen of an amount of energy equal to the energy burst that illuminated the screen. These points of discharge will be more likely to occur at those areas on the screen where the illumination is greatest. Over time we would get these dots of light filling the screen in the interference pattern.

We have a 'reciprocal relation' between 'energy' and 'time'. Thus, 'lowering energy intensity' while 'increasing time duration' is equivalent to 'increasing energy intensity' and 'lowering time duration'. But the resulting phenomenon is the same: the interference pattern we observe. This explanation of the double-slit experiment is logically consistent with the 'probability distribution' interpretation of Quantum Mechanics. The view we have of energy propagating continuously as a wave while manifesting locally in discrete units (equal size sips) when local equilibrium occurs, helps resolve the wave-particle dilemma.

\subsection{Explanation summary}

The argument presented above rests on the following ideas. These are consistent with all our results presented in this Chapter.

1. The 'electron emitted' is not be the same as the 'electron detected'.

2. Energy 'propagates continuously' but 'interacts discretely' when equilibrium occurs

3. We have 'accumulation of energy' before 'manifestation of energy'.

Our thinking and reasoning are also guided by the following attitude of physical realism:

a. Changing our detection devices while keeping the experimental setup the same can reveal something 'more' of the examined phenomenon but not something 'contradictory'.

b. If changing our detection devices reveals something 'contradictory', this is due to the detection device design and not to a change in the physics of the phenomenon examined.

Thus, using physical realism we argue that if we keep the experimental apparatus constant but only replace our 'detection devices' and as a consequence we detect something contradictory, the physics of the double slit experiment does not change. The experimental behavior has not changed, just the display of this behavior by our detection device has changed. The 'source' of the beam has not changed. The effect of the double slit barrier on that beam has not changed. So if our detector is now telling us that we are detecting 'particles' whereas before using other detector devices we were detecting 'waves', physical realism should tell us that this is entirely due to the change in our methods of detection. For the same input, our instruments may be so designed to produce different outputs.

\section{Conclusion}

In this Chapter we have sought to present a thumbnail sketch of a world without quanta. We started at the very foundations of Modern Physics with a simple and continuous mathematical derivation of Planck's Law. We demonstrated that Planck's Law is an exact mathematical identity that describes the interaction of energy. This fact alone explains why Planck's Law fits so exceptionally well the experimental data. 
Using our derivation of Planck's Law as a Rosetta Stone (linking Mechanics, Quantum Mechanics and Thermodynamics) we considered the quantity eta that naturally appears in our derivation as prime physis. Planck's constant $h$ is such a quantity. Energy can be defined as the time-rate of eta while momentum as the space-rate of eta. Other physical quantities can likewise be defined in terms of eta. Laws of Physics can and must be mathematically derived and not physically posited as Universal Laws chiseled into cosmic dust by the hand of God.

We postulated the Identity of Eta Principle, derived the Conservation of Energy and Momentum, derived Newton's Second Law of Motion, established the intimate connection between entropy and time, interpreted Schoedinger's equation and suggested that the wave-function $\psi$ is in fact prime physis $\eta$. We showed that The Second Law of Thermodynamics pertains to time (and not entropy, which can be both positive and negative) and should be reworded to state that 'all physical processes take some positive duration of time to occur'. We also showed the unexpected mathematical equivalence between Planck's Law and Boltzmann's Entropy Equation and proved that "if the speed of light is a constant, then light is a wave".

\section{Appendix: Mathematical derivations}

The proofs to many of the derivations below are too simple and are omitted for brevity. But the propositions are listed for purposes of reference and completeness of exposition.

Notation. We will consistently use the following notation throughout this APPENDIX:

$E(t)$ is a real-valued function of the real-variable $t$

$\Delta t=t-s$ is an 'interval of $\mathrm{t}^{\prime}$

$\Delta E=E(t)-E(s)$ is the 'change of $E^{\prime}$

$P=\int_{s}^{t} E(u) d u$ is the 'accumulation of $E^{\prime}$

$\bar{E}=E_{a v}=\frac{1}{t-s} \int_{s}^{t} E(u) d u$ is the 'average of $E^{\prime}$

$D_{x}$ indicates 'differentiation with respect to $x$ '

$r$ is a constant, often an 'exponential rate of growth'

\subsection{Part I: Exponential functions}

We will use the following characterization of exponential functions without proof:

Basic Characterization: $E(t)=E_{0} e^{r t}$ if and only if $D_{t} E=r E$

Characterization 1: $E(t)=E_{0} e^{r t}$ if and only if $\Delta E=\operatorname{Pr}$

Proof: Assume that $E(t)=E_{0} e^{r t}$. We have that $\Delta E=E(t)-E(s)=E_{0} e^{r t}-E_{0} e^{r s}$,

while $P=\int_{s}^{t} E_{0} e^{r u} d u=\frac{1}{r}\left[E_{0} e^{r t}-E_{0} e^{r s}\right]=\frac{\Delta E}{r}$. Therefore $\Delta E=\operatorname{Pr}$.

Assume next that $\Delta E=\operatorname{Pr}$. Differentiating with respect to $t, D_{t} E=r D_{t} P=r E$.

Therefore by the Basic Characterization, $E(t)=E_{0} e^{r t}$. q.e.d 
Theorem 1: $E(t)=E_{0} e^{r t}$ if and only if $\frac{P r}{e^{r \Delta t}-1}$ is invariant with respect to $t$

Proof: Assume that $E(t)=E_{0} e^{r t}$. Then we have, for fixed s,

$$
P=\int_{s}^{t} E_{0} e^{r u} d u=\frac{E_{0}}{r}\left[e^{r t}-e^{r s}\right]=\frac{E_{0} e^{r s}}{r}\left[e^{r(t-s)}-1\right]=\frac{E(s)}{r}\left(e^{r(t-s)}-1\right)
$$

and from this we get that $\frac{P r}{e^{r \Delta t}-1}=E(s)=$ constant. Assume next that $\frac{P r}{e^{r \Delta t}-1}=C$ is constant with respect to $t$, for fixed s.

Therefore, $D_{t}\left[\frac{P r}{e^{r \Delta t}-1}\right]=\frac{r E(t) \cdot\left[e^{r \Delta t}-1\right]-r P \cdot\left[r e^{r \Delta t}\right]}{\left(e^{r \Delta t}-1\right)^{2}}=0$ and so, $E(t)=\left(\frac{P r}{e^{r \Delta t}-1}\right) e^{r \Delta t}=C \cdot e^{r \Delta t}$ where $C$ is constant. Letting $t=s$ we get $E(s)=C$. We can rewrite this as $E(t)=E(s) e^{r(t-s)}=E_{0} e^{r t}$. q.e.d

From the above, we have

Characterization 2: $E(t)=E_{0} e^{r t}$ if and only if $\frac{P r}{e^{r(t-s)}-1}=E(s)$

Clearly by definition of $E_{a v}, r \Delta t=\frac{P r}{E_{a v}}$. We can write $\frac{P r}{e^{r \Delta t}-1}$ equivalently as $\frac{P r}{e^{P r / E_{a v}}-1}$ in the above. Theorem 1 above can therefore be restated as,

Theorem 1a: $E(t)=E_{0} e^{r t}$ if and only if $\frac{P r}{e^{P r / E_{a v}}-1}$ is invariant with $t$

The above Characterization 2 can then be restated as

Characterization 2a: $E(t)=E_{0} e^{r t}$ if and only if $\frac{P r}{e^{P r / E_{a v}}-1}=E(s)$.

But if $\frac{P r}{e^{P r / E_{a v}}-1}=E(s)$, then by Characterization $2 a, E(t)=E_{0} e^{r t}$. Then, by Characterization 1, we must have that $\Delta E=\operatorname{Pr}$. And so we can write equivalently $\frac{\Delta E}{e^{\Delta E / E_{a v}}-1}=E(s)$. We have the following equivalence,

Characterization 3: $E(t)=E_{0} e^{r t}$ if and only if $\frac{\Delta E}{e^{\Delta E / E_{a v}}-1}=E(s)$

As we've seen above, it is always true that $\frac{P r}{E_{a v}}=r \Delta t$. But for exponential functions $E(t)$ we also have that $\Delta E=\operatorname{Pr}$. So, for exponential functions we have the following.

Characterization 4: $E(t)=E_{0} e^{r t} \quad$ if and only if $\quad \frac{\Delta E}{E_{a v}}=r \Delta t$

\subsection{Part II: Integrable functions}

We next consider that $E(t)$ is any function. In this case, we have the following. 
Theorem 2: a) For any differentiable function $E(t), \lim _{t \rightarrow s} \frac{\Delta E}{e^{\Delta E / E_{a v}}-1}=E(s)$

b) For any integrable function $E(t), \quad \lim _{t \rightarrow s} \frac{P r}{e^{r \Delta t}-1}=E(s)$

Proof: Since $\frac{\Delta E}{e^{\Delta E / E_{a v}}-1} \rightarrow \frac{0}{0}$ and $\frac{P r}{e^{r \Delta t}-1} \rightarrow \frac{0}{0}$ as $t \rightarrow s$, we apply L'Hopital's Rule.

$$
\begin{gathered}
\lim _{t \rightarrow s} \frac{\Delta E}{e^{\Delta E / \bar{E}}-1}=\lim _{t \rightarrow s} \frac{D_{t} E(t)}{e^{\Delta E / \bar{E}} \cdot\left[\frac{D_{t} E(t) \cdot \bar{E}-D_{t} \bar{E} \cdot \Delta E}{\bar{E}^{2}}\right]} \\
\quad=\lim _{t \rightarrow s} \frac{\bar{E}^{2} \cdot D_{t} E(t)}{e^{\Delta E / \bar{E}} \cdot\left[D_{t} E(t) \cdot \bar{E}-D_{t} \bar{E} \cdot \Delta E\right]}=E(s)
\end{gathered}
$$

since $\Delta E \rightarrow 0$ and $\bar{E} \rightarrow E(s)$ as $t \rightarrow s$.

Likewise, we have $\lim _{t \rightarrow s} \frac{P r}{e^{r \Delta t}-1}=\lim _{t \rightarrow s} \frac{E(s) r}{e^{r \Delta t} \cdot r}=E(s)$. q.e.d.

Corollary $A: \frac{\Delta E}{e^{\Delta E / \bar{E}}-1}$ is invariant with $t$ if and only if $E(s)=\frac{\Delta E}{e^{\Delta E / \bar{E}}-1}$

Proof: Using Theorem 2 we have $\lim _{t \rightarrow s} \frac{\Delta E}{e^{\Delta E / E_{a v}}-1}=E(s)$. Since $\frac{\Delta E}{e^{\Delta E / E_{a v}}-1}$ is constant with respect to $t$, we have $E(s)=\frac{\Delta E}{e^{\Delta E / E_{a v}}-1}$. Conversely, if $E(s)=\frac{\Delta E}{e^{\Delta E / E_{a v}}-1}$, then by Characterization 3, $E(s)=E_{0} e^{r s}$. Since $E(s)$ is a constant, $\frac{\Delta E}{e^{\Delta E / E_{a v}}-1}$ is invariant with respect to t.q.e.d

Since it is always true by definitions that $r \Delta t=\frac{P r}{E_{a v}}$, Theorem 2 can also be written as,

Theorem 2a: For any integrable function $E(t), \lim _{t \rightarrow s} \frac{\operatorname{Pr}}{e^{\operatorname{Pr} / E_{a v}}-1}=E(s)$

As a direct consequence of the above, we have the following interesting and important result: Corollary B: $E(s)=\frac{\Delta E}{e^{\Delta E / E_{a v}}-1}$ and $E(s)=\frac{P r}{e^{P r / E_{a v}}-1}$ are independent of $\Delta t, \Delta E$.

\subsection{Part III: Independent proof of Characterization 3}

In the following we provide a direct and independent proof of Characterization 3 .

We first prove the following,

Lemma: For any $E, D_{t} \bar{E}(t)=\frac{E(t)-\bar{E}}{t-s}$ and $D_{s} \bar{E}(s)=\frac{\bar{E}-E(s)}{t-s}$

Proof: We let $\Delta t=t-s$ and $\bar{E}=\frac{1}{t-s} \int_{s}^{t} E(u) d u$.

Differentiating with respect to $t$ we have $(t-s) \cdot D_{t} \bar{E}(t)+\bar{E}=E(t)$. 
Rewriting, we have $D_{t} \bar{E}(t)=\frac{E(t)-\bar{E}}{t-s}$. Differentiating with respect to $s$ we have $(t-s) \cdot D_{s} \bar{E}(s)-\bar{E}=-E(s)$. Rewriting, we have $D_{s} \bar{E}(s)=\frac{\bar{E}-E(s)}{t-s}$. q.e.d.

Characterization 3: $E(t)=E_{0} e^{r t}$ if and only if $\frac{\Delta E}{e^{\Delta E / E_{a v}}-1}=E(s)$

Proof: Assume that $E(t)=E_{0} e^{r t}$. From,

$$
P=\int_{s}^{t} E_{0} e^{r u} d u=\frac{E_{0}}{r}\left[e^{r t}-e^{r s}\right]=\frac{E_{0} e^{r s}}{r}\left[e^{r \Delta t}-1\right]=\frac{E(s)}{r}\left[e^{r \Delta t}-1\right]
$$

we get, $E(s)=\frac{P r}{e^{r \Delta t}-1}$. This can be rewritten as, $E(s)=\frac{P r}{e^{P r / E_{a v}}-1}$. Since $\Delta E=\operatorname{Pr}$, this can further be written as $E(s)=\frac{\Delta E}{e^{\Delta E / E_{a v}}-1}$.

Conversely, consider next a function $E(s)$ satisfying

$$
E(s)=\frac{\Delta E}{e^{\xi}-1}, \text { where }\left\{\begin{array}{l}
\Delta E=E(t)-E(s) \\
\Delta t=t-s \\
\xi=\frac{\Delta E}{\bar{E}} \\
\bar{E}=\frac{1}{\Delta t} \int_{s}^{t} E(u) d u
\end{array} \text { and } t\right. \text { can be any real value. }
$$

From the above, we have that $e^{\xi}=\frac{\Delta E}{E(s)}+1=\frac{E(t)-E(s)+E(s)}{E(s)}=\frac{E(t)}{E(s)}$.

Differentiating with respect to $s$, we get $e^{\xi} \cdot D_{s} \xi=\frac{-E(t) \cdot D_{s} E(s)}{E(s)^{2}}=-e^{\xi} \cdot \frac{D_{s} E(s)}{E(s)}$

and so, $D_{s} \xi=-\frac{D_{s} E(s)}{E(s)}$

From the above Lemma we have

$$
D_{s} \bar{E}(s)=\frac{\bar{E}-E(s)}{t-s}
$$

Differentiating $\xi=\frac{\Delta E}{\bar{E}}$ with respect to s we get,

$$
D_{s} \xi=\frac{-D_{s} E(s) \cdot \bar{E}-\Delta E \cdot D_{s} \bar{E}(s)}{\bar{E}^{2}}
$$

and combining (A1), (A2), and (A3) we have

$$
-\frac{D_{s} E(s)}{E(s)}=\frac{-D_{s} E(s) \cdot \bar{E}-\frac{\Delta E}{\Delta t}(\bar{E}-E(s))}{\bar{E}^{2}}=-\frac{D_{s} E(s)}{\bar{E}}-\frac{\Delta E}{\Delta t} \cdot \frac{(\bar{E}-E(s))}{\bar{E}^{2}}
$$


We can rewrite the above as follows,

$$
\frac{D_{s} E(s)}{E(s)}-\frac{D_{s} E(s)}{\bar{E}}=D_{s} E(s)\left(\frac{\bar{E}-E(s)}{E(s) \cdot \bar{E}}\right)=\frac{\Delta E}{\Delta t} \cdot \frac{(\bar{E}-E(s))}{\bar{E}^{2}}
$$

and so, $\quad \frac{D_{s} E(s)}{E(s)}=\frac{\Delta E}{\Delta t} \cdot \frac{1}{\bar{E}}$.

Using (A1), this can be written as

$$
-D_{s} \xi=\frac{\xi}{\Delta t}, \text { or as } \xi=-D_{s} \xi \cdot \Delta t
$$

Differentiating (A4) above with respect to $s$, we get $D_{s} \xi=-D_{s}^{2} \xi \cdot \Delta t+D_{s} \xi$.

Therefore, $D_{s}^{2} \xi=0$. Working backward, this gives $D_{s} \xi=-r=$ constant.

From (A1), we then have that $\frac{D_{s} E(s)}{E(s)}=r$ and therefore $E(s)=E_{0} e^{r s}$. q.e.d.

\section{Acknowledgement}

I am indebted to Segun Chanillo, Prof. of Mathematics, Rutgers University for his encouragement, when all others thought my efforts were futile. Also, I am deeply grateful to Hayrani Oz, Prof. of Aerospace Engineering, Ohio State University, who discovered my posts on the web and was the first to recognize the significance of my results in Physics. Special thanks also to Miguel Bayona of The Lawrenceville School for his friendship and help with the graphics in this chapter. And Alexander Morisse who is my best and severest critic of the Physics in these results.

\section{References}

Frank, Adam (2010) , Who Wrote the Book of Physics? Discover Magazine (April 2010)

Keesing, Richard (2001). Einstein, Millikan and the Photoelectric Effect, Open University Physics Society Newsletter, Winter 2001/2002 Vol 1 Issue 4 http://www.oufusion.org.uk/pdf/FusionNewsWinter01.pdf

Öz, H., Algebraic Evolutionary Energy Method for Dynamics and Control, in: Computational Nonlinear Aeroelasticity for Multidisciplinary Analysis and Design, AFRL, VA-WP-TR2002 -XXXX, 2002, pp. 96-162.

Öz, H., Evolutionary Energy Method (EEM): An Aerothermoservoelectroelastic Application,: Variational and Extremum Principles in Macroscopic Systems, Elsevier, 2005, pp. 641-670.

Öz , H., The Law Of Evolutionary Enerxaction and Evolutionary Enerxaction Dynamics, Seminar presented at Cambridge University, England, March 27, 2008, http://talks.cam.ac.uk/show/archive/12743

Öz , Hayrani; John K. Ramsey, Time modes and nonlinear systems, Journal of Sound and Vibration, 329 (2010) 2565-2602, doi:10.1016/j.jsv.2009.12.021

Planck, Max (1901) On the Energy Distribution in the Blackbody Spectrum, Ann. Phys. 4, 553, 1901

Ragazas, C. (2010) A Planck-like Characterization of Exponential Functions, knol 
http:/ / knol.google.com/k/constantinos-ragazas/a-planck-like-characterizationof/q147o1qdr604/7\#

Ragazas, C. (2010) Entropy and 'The Arrow of Time', knol http://knol.google.com/k/ constantinos-ragazas/entropy-and-the-arrow-of-time/q147o1qdr604/17\#

Ragazas, C. (2010) "Let there be $h "$ ": An Existance Argument for Planck's Constant, knol http://knol.google.com/k/constantinos-ragazas/let-there-be-h-an-existenceargument/q147o1qdr604/12\#

Ragazas, C. (2010) Prime 'physis' and the Mathematical Derivation of Basic Law, knol http://knol.google.com/k/constantinos-ragazas/prime-physis-and-themathematical/q147o1qdr604/10\#

Ragazas, C. (2010) "The meaning of $\psi^{\prime \prime}$ : An Interpretation of Schroedinger's Equations, knol http://knol.google.com/k/constantinos-ragazas/the-meaning-of-psi-aninterpretation-of/q147o1qdr604/14\#

Ragazas, C. (2010) Planck's Law is an Exact Mathematical Identity, knol http:/ / knol.google.com/k/constantinos-ragazas/planck-s-law-is-an-exactmathematical/q147o1qdr604/3\#

Ragazas, C. (2010) The Temperature of Radiation, knol http://knol.google.com/k /constantinos-ragazas/the-temperature-of-radiation/q147o1qdr604/6\#

Ragazas, C. (2010) The Interaction of Measurement, knol http://knol.google.com/k/ constantinos-ragazas/the-interaction-of-measurement/q147o1qdr604/11\#

Ragazas, C. (2010) A Time-dependent Local Representation of Energy, knol http:// knol.google.com/k/constantinos-ragazas/a-time-dependent-localrepresentation/q147o1qdr604/9\#

Ragazas, C. (2010)A Plausable Explanation of the Double-slit Experiment in Physics, knol http://knol.google.com/k/constantinos-ragazas/a-plausible-explanation-of-thedouble/q147o1qdr604/4\#

Ragazas, C. (2010) The Photoelectric Effect Without Photons, knol http://knol.google.com/k/ constantinos-ragazas/the-photoelectric-effect-without-photons/q147o1qdr604/8\#

Ragazas, C. (2010) Stocks and Planck's Law, knol http://knol.google.com/k/constantinosragazas/stocks-and-planck-s-law/q147o1qdr604/2\#

Ragazas, C. (2011) What is The Matter With de Broglie Waves? knol http:/ / knol.google.com/k/constantinos-ragazas/what-is-the-matter-with-debroglie-waves/q147o1qdr604/18\#

Ragazas, C. (2011) "If the Speed of Light is a Constant, Then Light is a Wave", knol http:/ / knol.google.com/k/constantinos-ragazas/if-the-speed-of-light-is-aconstant/ql47o1qdr604/19\#

Tonomura (1989) http:/ / www.hitachi.com/rd/research/em/doubleslit.html

Wikipedia, (n.d.) http://en.wikipedia.org/wiki/File:Firas_spectrum.jpg 


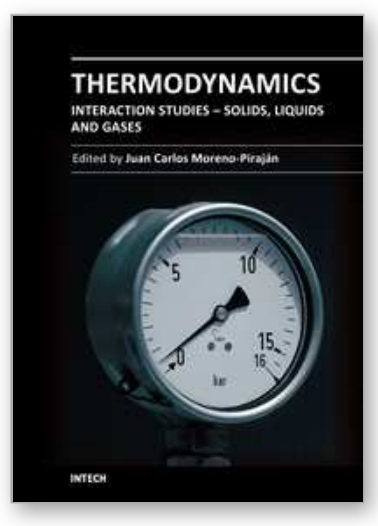

\author{
Thermodynamics - Interaction Studies - Solids, Liquids and Gases \\ Edited by Dr. Juan Carlos Moreno Pirajễin
}

ISBN 978-953-307-563-1

Hard cover, 918 pages

Publisher InTech

Published online 02, November, 2011

Published in print edition November, 2011

Thermodynamics is one of the most exciting branches of physical chemistry which has greatly contributed to the modern science. Being concentrated on a wide range of applications of thermodynamics, this book gathers a series of contributions by the finest scientists in the world, gathered in an orderly manner. It can be used in post-graduate courses for students and as a reference book, as it is written in a language pleasing to the reader. It can also serve as a reference material for researchers to whom the thermodynamics is one of the area of interest.

\title{
How to reference
}

In order to correctly reference this scholarly work, feel free to copy and paste the following:

Constantinos Ragazas (2011). The Thermodynamics in Planck's Law, Thermodynamics - Interaction Studies Solids, Liquids and Gases, Dr. Juan Carlos Moreno PirajÃ in (Ed.), ISBN: 978-953-307-563-1, InTech, Available from: http://www.intechopen.com/books/thermodynamics-interaction-studies-solids-liquids-andgases/the-thermodynamics-in-planck-s-law

\section{INTECH}

open science | open minds

\section{InTech Europe}

University Campus STeP Ri

Slavka Krautzeka 83/A

51000 Rijeka, Croatia

Phone: +385 (51) 770447

Fax: +385 (51) 686166

www.intechopen.com

\section{InTech China}

Unit 405, Office Block, Hotel Equatorial Shanghai

No.65, Yan An Road (West), Shanghai, 200040, China

中国上海市延安西路65号上海国际贵都大饭店办公楼405单元

Phone: +86-21-62489820

Fax: $+86-21-62489821$ 
(C) 2011 The Author(s). Licensee IntechOpen. This is an open access article distributed under the terms of the Creative Commons Attribution 3.0 License, which permits unrestricted use, distribution, and reproduction in any medium, provided the original work is properly cited. 